\title{
Rapid Discrimination of Chlorpheniramine Maleate and Assessment of Its Surface Content Uniformity in a Pharmaceutical Formulation by NIR-CI Coupled with Statistical Measurement
}

\author{
Luwei Zhou, ${ }^{1,2,3,4}$ Zhisheng Wu, ${ }^{1,2,3,4}$ Xinyuan Shi, ${ }^{1,2,3,4}$ Manfei Xu, ${ }^{1,2,3,4}$ Xiaona Liu, ${ }^{1,2,3,4}$ \\ Bing Xu, ${ }^{1,2,3,4}$ and Yanjiang Qiao ${ }^{1,2,3,4}$ \\ ${ }^{1}$ Beijing University of Chinese Medicine, Beijing 100102, China \\ ${ }^{2}$ Pharmaceutical Engineering and New Drug Development of Traditional Chinese Medicine (TCM) of Ministry of Education, \\ Beijing 100102, China \\ ${ }^{3}$ Key Laboratory of TCM-Information Engineering of State Administration of TCM, Beijing 100102, China \\ ${ }^{4}$ Beijing Key Laboratory for Basic and Development Research on Chinese Medicine, Beijing 100102, China
}

Correspondence should be addressed to Zhisheng Wu; wzs@bucm.edu.cn and Yanjiang Qiao; yjqiao@263.net

Received 6 March 2014; Revised 31 May 2014; Accepted 2 June 2014; Published 26 June 2014

Academic Editor: Kong-Thon Tsen

Copyright (C) 2014 Luwei Zhou et al. This is an open access article distributed under the Creative Commons Attribution License, which permits unrestricted use, distribution, and reproduction in any medium, provided the original work is properly cited.

This study demonstrated that near infrared chemical imaging (NIR-CI) was a rapid and nondestructive technique for discrimination of chlorpheniramine maleate (CPM) and assessment of its surface content uniformity (SCU) in a pharmaceutical formulation. The characteristic wavenumber method was used for discriminating CPM distribution on the tablet surface. To assess the surface content uniformity of CPM, binary image and statistical measurement were proposed. Furthermore, high-performance liquid chromatography (HPLC) was used as reference method for accurately determining volume content of CPM in the sample. Moreover, HPLC was performed to assess volume content uniformity (VCU) of CPM in whole region and part region of the tablets. The NIRCI result showed that the spatial distribution of CPM was heterogeneous on the tablet surface. Through the comparison of content uniformity of CPM determined by NIR-CI and HPLC, respectively, it demonstrated that a high degree of VCU did not imply a high degree of SCU of the samples. These results indicate that HPLC method is not suitable for testing SCU, and this has been verified by NIR-CI. This study proves the feasibility of NIR-CI for rapid discrimination of CPM and assessment of its SCU, which is helpful for the quality control of commercial CPM tablets.

\section{Introduction}

The discrimination of active ingredient is essential for drug analysis because counterfeit drug products not only threaten the public health but also damage the finance and reputation of legitimate pharmaceutical companies. According to the definition of counterfeit drug by World Health Organization (WHO), the counterfeit products contain wrong ingredients, without active ingredients or with insufficient active ingredients [1]. Furthermore, ingredients uniformity analysis of drug products has also been proposed by pharmaceutical regulations. Content uniformity will affect the speed of disintegration and dissolution and further influence the in vivo bioavailability and the efficacy. Recently, near infrared chemical imaging (NIR-CI) has shown large potential in quality control of legitimate pharmaceutical products.

So far, a variety of destructive methods, including highperformance liquid chromatography (HPLC) [2, 3] and mass spectrometry (MS) [4], are frequently used to discriminate active pharmaceutical ingredients (APIs). In contrast, vibrational spectroscopy techniques show rapid and nondestructive advantages. Vibrational spectroscopy, such as near-infrared (NIR) spectroscopy and Raman spectroscopy, is applied for detecting the chemical compositions and physical properties. Several studies have been published for 
discriminating active ingredients [5-8]. However, vibrational spectroscopy is used for discriminating API and determining its content without spatial information. Thus, NIR-CI adds a new dimension to NIR spectroscopy, which means that the spatial distributions of APIs could be visualized.

Palou et al. reported that content and spatial distributions of major and minor ingredients were determined during the development of a new pharmaceutical formulation. In addition, the thickness and the surface distribution of the coating film were also measured [9]. Ma and Anderson determined blend homogeneity of ternary mixtures using NIR-CI technology. The results demonstrated a good correlation between NIR-CI data and ultraviolet-visible spectrophotometry (UV-Vis) data [10]. Moreover, the content uniformity of acetylsalicylic acid (ASA) commercial tablets of four different brands was assessed using NIR-CI. The ASA contents determined by NIR-CI technique were close to the nominal contents used as reference. Finally, the result demonstrated that the content uniformity of each brand satisfied the requirement of the European Pharmacopoeia [11]. Dubois et al. described NIR-CI instrument equipped with a focal plane array detector for counterfeit drug identification. A total of 30 tablets, including 10 genuine tablets and 20 counterfeit tablets, were investigated and the counterfeit tablets were successfully identified [12]. Certainly, there are other pharmaceutical applications of NIR-CI technique, which can refer to previous reviews $[13,14]$.

However, only destructive methods (i.e., HPLC) and vibrational spectroscopy (NIR, Raman) are developed for identifying the ingredients and assessing the content uniformity in Chinese pharmaceutical quality standards. With the increasing acceptance of the concepts of process analytical technology (PAT) [15] and quality by design (QbD) [16] in pharmaceutical industry, the novel NIR-CI technique should be applied to pharmaceutical quality assurance in China. Compared with wet analytical methods, the implementation of NIR-CI for ingredients discrimination will improve the economic efficiency (fast and nondestructive) and protect the environment (without sample preparation).

Chlorpheniramine maleate (CPM), which is an $\mathrm{H} 1$ receptor antagonist, has been clinically used to alleviate symptoms of cold and treat the allergic diseases. CPM has a strong action of antihistamine, which could cause drowsiness and restrain the central system [17]. Due to the good therapeutic effect and cheap price, CPM products are popular among patients in China. There are 434 pharmaceutical industries which produce CPM products in China (with the approval of China Food and Drug Administration). In order to ensure the quality of pharmaceutical products, it is necessary to discriminate API and assess its content uniformity. Therefore, CPM tablets were chosen as examples in this study. NIR-CI was performed to discriminate CPM and assess its surface content uniformity (SCU).

Despite the superiorities of NIR-CI, the acquired data is a three-dimensional hypercube. To extract related information, chemometric methods should be performed for discrimination purpose, such as basic analysis of correlation between analytes (BACRA) [18, 19] and characteristic wavenumber method. Besides, image analysis methods (i.e., binary image)
[20] coupled with statistical measurement are also indispensable for assessing the surface content uniformity of CPM. In addition, NIR-CI as a novel technique, HPLC was used as reference method to determine the volume content of CPM in the sample and access its volume content uniformity (VCU). The advantages of NIR-CI were highlighted in comparison with HPLC method. This study showed that NIRCI technology coupled with statistical measurement could be recommended in surface content uniformity assessment of commercial CPM tablets.

\section{Materials and Methods}

2.1. Materials. The chlorpheniramine maleate was purchased from Haohua Industry Corporation (Jinan, China). The excipients of the CPM tablet were pregelatinized starch (Colorcon, USA), microcrystalline cellulose (Beijing Fengli Jingqiu Commerce and Trade Corporation, China), and magnesium stearate (Sinopharm Chemical Reagent Corporation, China). Standard CPM was obtained from National Institutes for Food and Drug Control (Beijing, China). Acetonitrile and phosphoric acid were purchased from Fisher Scientific (chromatographic grade, USA). Ammonium dihydrogen phosphate was provided by Beijing Chemical Works (analytical grade, China). Deionized water was purified by Milli-Q water system (Millipore Corporation, USA).

\subsection{Pilot-Scale Production of Chlorpheniramine Maleate} Tablets. The CPM tablets were from pilot-scale manufacture by us (Good Manufacturing Practice (GMP) standard and Pharmaceutical Engineering and New Drug Development of Traditional Chinese Medicine of Ministry of Education). The homogeneous powder containing active pharmaceutical ingredient and excipients was produced in a blender using the equal incremental method according to the proportions of prescription (CPM, 4\% w/w; pregelatinized starch, 50\% $\mathrm{w} / \mathrm{w}$; microcrystalline cellulose, $45.5 \% \mathrm{w} / \mathrm{w}$; and magnesium stearate, $0.5 \% \mathrm{w} / \mathrm{w})$. The dry-blend mixtures were compressed into $1.0 \mathrm{~g}$ tablets under the parameters of compression pressure $60 \mathrm{KN}$, the depth of filling material $10.0 \mathrm{~mm}$, and the thickness of the tablets $2.0 \mathrm{~mm}$ (pilot-scale rotary tablet press, Xinyuan Pharmaceutical Machinery Corporation, Shanghai, China). A flat punch set was utilized to acquire a flat sample surface. Pure compound reference tablets of CPM and major excipients (pregelatinized starch and microcrystalline cellulose) were also produced under the same parameters.

\subsection{HPLC Method for the Determination of Chlorpheniramine} Maleate. The content of CPM in the entire volume of the sample was determined by RP-HPLC method recommended by the Chinese Pharmacopoeia (ChP 2010 edition, volume II). An Agilent 1100 HPLC system (Agilent Technologies, USA) with a quaternary pump, a vacuum degasser, a thermostatic column compartment, an autosampler, and a diode array detector (DAD) detector was utilized. Separation was performed on Spursil C18 column with a particle size of $5 \mu \mathrm{m}$ (250 mm $\times 4.6 \mathrm{~mm}$, Dikma Technologies, Beijing, China) at $30^{\circ} \mathrm{C}$. The mobile phase consisted of deionized water with $0.1 \%$ phosphoric acid and acetonitrile $(80: 20, \mathrm{v} / \mathrm{v})$. The flow 
rate was maintained at $1.0 \mathrm{~mL} / \mathrm{min}$ and the $\mathrm{UV}$ signal was monitored at $262 \mathrm{~nm}$.

Ten tablets were selected and each of them was dissolved by mobile phase. In addition, another ten tablets from the same pilot-scale manufacture batch were selected and split into four equal parts. Each part was dissolved by mobile phase properly. Then the diluent was filtered through a $0.45 \mu \mathrm{m}$ Millipore filter membrane, and $10 \mu \mathrm{L}$ filtrate was injected into the HPLC system for determination.

\subsection{Near-Infrared Chemical Imaging}

2.4.1. Data Acquisition and Transformation. The tablet was fixed onto the microscope slide and measured directly on the surface of tablet. The images of different regions on the tablet surface were collected to get spatial information of different regions and compare the differentiations. Sample was analyzed on Spotlight 400 N FT-NIR Imaging Systems equipped with a linear mercury cadmium telluride (MCT) array detector (PerkinElmer, UK). An area of $2000 \mu \mathrm{m} \times$ $2000 \mu \mathrm{m}$ was imaged using spectrum resolution $16 \mathrm{~cm}^{-1}$ and spatial resolution $25 \mu \mathrm{m} \times 25 \mu \mathrm{m}$ (pixel size) thus acquiring a total of 6400 spectra for each image. Each spectrum was the average of 16 scans from wavenumber region $7800 \mathrm{~cm}^{-1}$ to $4000 \mathrm{~cm}^{-1}$. The total time of image acquisition was about 25 minutes.

Prior to sample scanning, it is necessary to correct the instrument response using a background reference. Thus, the raw data obtained by NIR line mapping system is the relative NIR diffuse reflectance data $\left(R=R_{\text {sample }} / R_{\text {background }}\right)$. The high reflectance standard Spectralon (Labsphere Inc., North Sutton, New Hampshire) was utilized as a background. Prior to data analysis, all relative diffuse reflectance data were transferred into absorbance data using the formula $A=$ $-\log _{10}(1 / R)$. Pure compound reference tablets of CPM and major excipients were also imaged to acquire pure spectra using the same conditions.

2.4.2. Data Processing. The data acquired by NIR-CI is threedimensional hypercube using the expression $M=(X \times$ $Y \times \lambda$ ), where $X$ and $Y$ are the spatial dimensions and $\lambda$ represents spectral information. The hyperspectral data could be analyzed by both the three-way methodology and the twoway methodology but two-way methods are more suitable for this type of data [21]. Three-dimensional matrix is unfolded to obtain the two-dimensional matrix $(X Y \times \lambda)$ and two-way approaches can be used. Then, the two-dimensional matrix is refolded to retain the spatial location of each pixel and generate the chemical image. In this study, hypercube was unfolded and ordinary two-way methods were utilized.

Prior to performing any chemometric methods that generate the chemical image, it is necessary to preprocess the spectra data in order to avoid the influence of unwanted phenomena frequently found in NIR measurements. Some pretreatment methods were performed, such as normalizing and Savitzky-Golay (SG) smoothing [22].

The characteristic wavenumber method is a univariate methodology that generates the chemical image of an ingredient at one specific wavenumber. Therefore, it is significant to

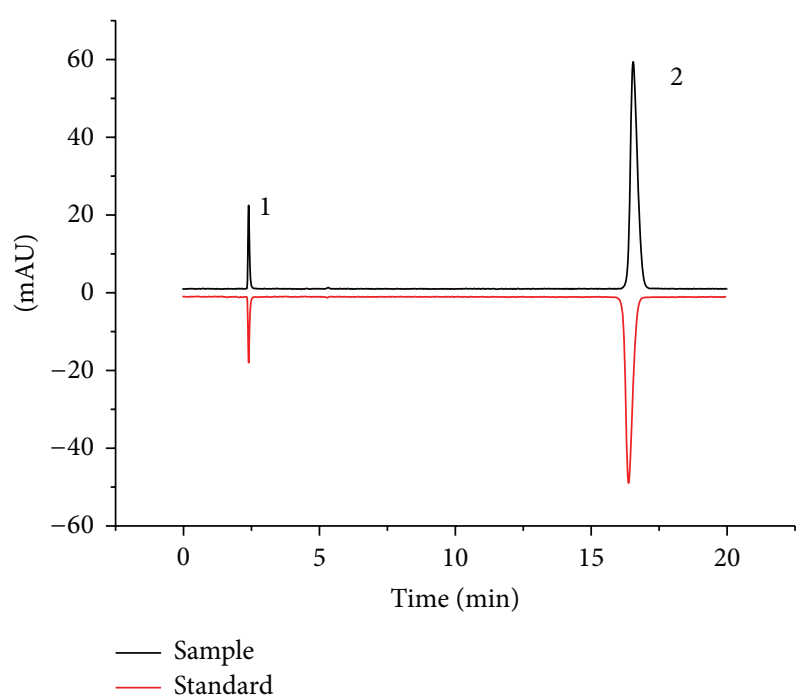

FIGURE 1: Chlorpheniramine maleate chromatogram.

select a wavelength with a sharp and distinct absorption for that specific ingredient, which has little spectral absorption interference from other ingredients in the sample. NIR spectra of pure ingredients are the most suitable for selecting the distinct absorption. Moreover, a derivative of NIR spectra is helpful to distinguish the overlapping peaks and improve the spectral resolution. In this study, pure component spectra preprocessed by SG smoothing and first derivative were utilized to select distinct absorption peak of CPM.

HyperView software and Spectrum Image software (PerkinElmer, UK) were used for data processing and analysis. Other data analysis was performed by homemade routines programmed in MATLAB software (MATLAB2009b, Mathworks, USA).

\section{Results and Discussion}

3.1. HPLC Method for the Determination of Chlorpheniramine Maleate. HPLC was utilized as reference method for accuracy determination of CPM in the entire volume of the sample. Since the HPLC method used here is recommended by the ChP (2010 edition, volume II), the accuracy and reproducibility of the method have already been approved officially. CPM chromatogram was shown in Figure 1.

The first peak and second peak represented maleate and chlorpheniramine, respectively. Chlorpheniramine peak area was calculated for volume content determination. The retention time of chlorpheniramine in the sample was the same with the standard. The linearity test for the quantitation of chlorpheniramine was carried out over the range of $0.2031-2.0310 \mu \mathrm{g}$. The parameters of the calibration curve $y=892.8 x-13.06\left(R^{2}=0.9999\right)$ for chlorpheniramine demonstrated a good linear relationship.

3.2. Pure Compound Images and Spectra. Each pure NIR image (CPM, pregelatinized starch, and microcrystalline cellulose) was collected and three absorbance spectra of the major compounds were obtained from the pure images. As 


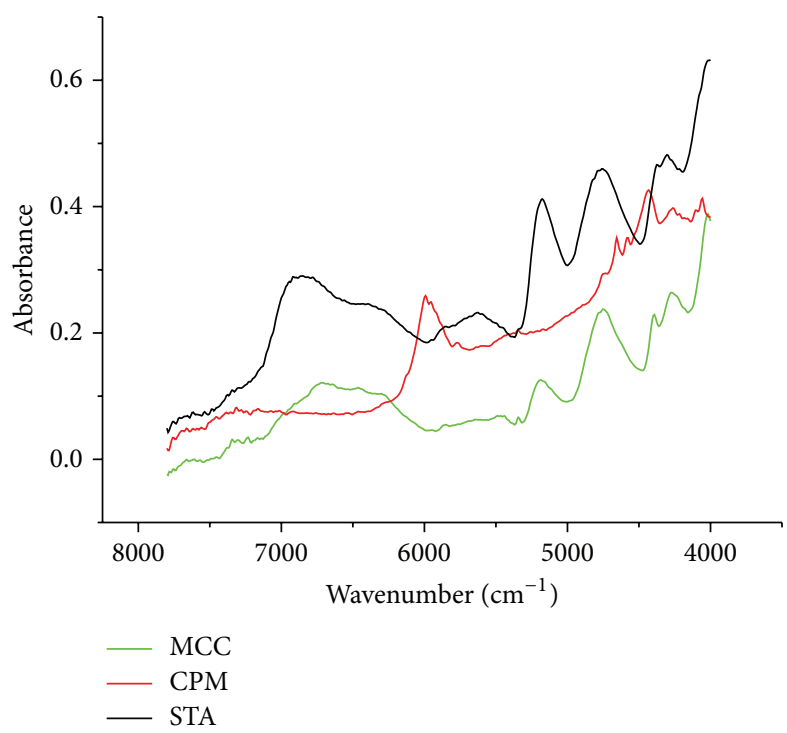

(a)

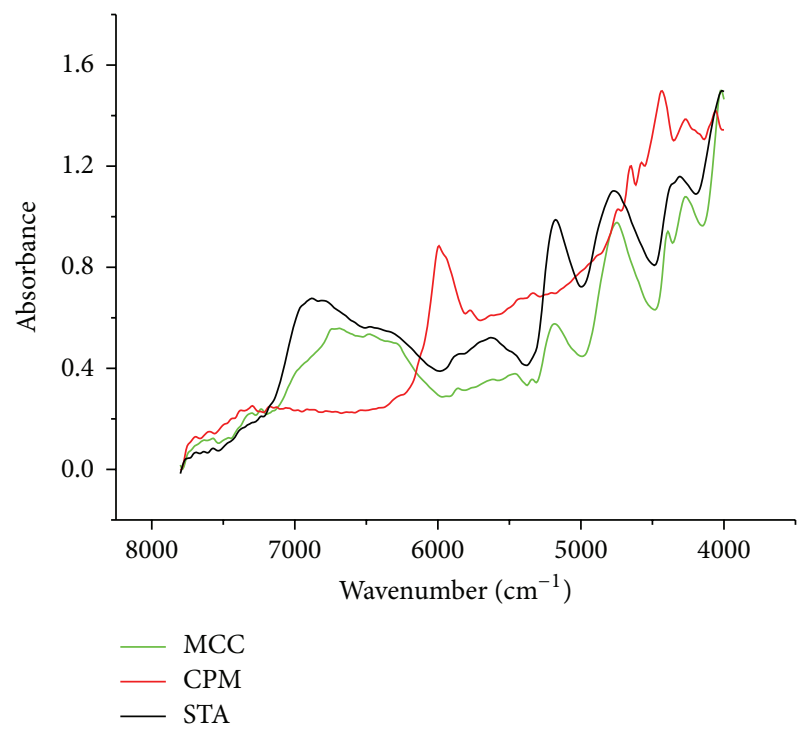

(b)

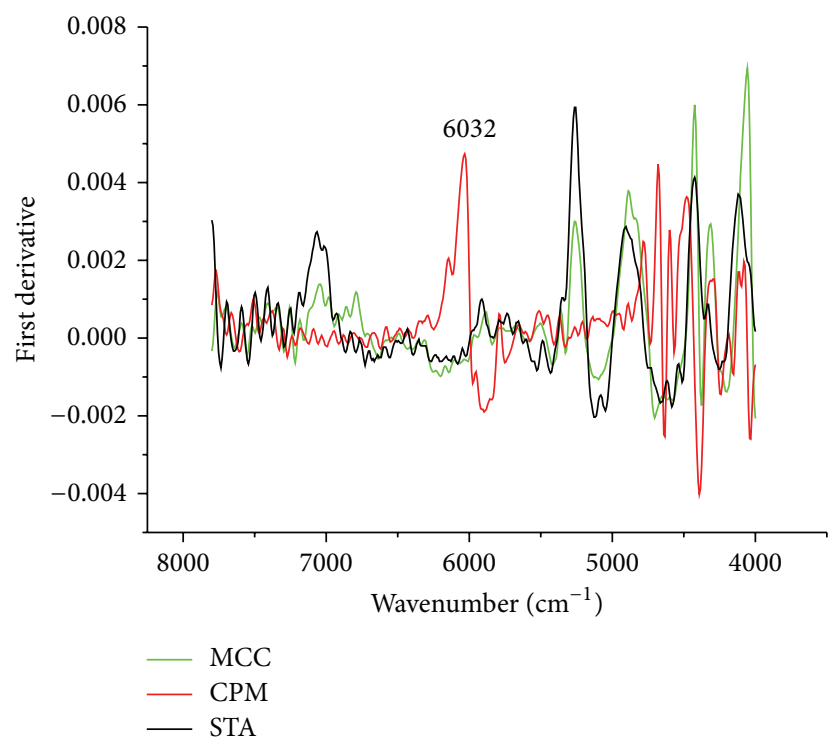

(c)

FIGURE 2: Raw and preprocessed spectra. (a) Raw spectra, (b) spectra preprocessed by SG smoothing and normalizing, and (c) spectra preprocessed by SG smoothing and first derivative of three major ingredients (CPM, pregelatinized starch (STA), and microcrystalline cellulose (MCC)).

Figure 2(a) (CPM) showed, there was large signal fluctuation in the spectral region of $5500 \mathrm{~cm}^{-1}-6500 \mathrm{~cm}^{-1}$. This spectral region contained main information, which could reflect the CPM chemical message in the samples. Moreover, all spectra showed broader absorbance and it was difficult to discriminate them from each other. An approach called basic analysis of correlation between analytes (BACRA) was proposed to clarify the differences between any two pure spectra.

\subsection{Rapid Discrimination of Chlorpheniramine Maleate on the Tablet Surface}

3.3.1. Basic Analysis of Correlation between Analytes (BACRA). BACRA method, based on the parameter of the Pearson correlation index, was proposed to distinguish the spectra. The correlation coefficients were calculated between the pure spectrum (CPM, pregelatinized starch, and microcrystalline cellulose) and each spectrum obtained from the corresponding single pixel on the surface of pure compound tablet. The correlation-coefficient images were obtained as Figure 3 showed. High correlation coefficients (above 0.95) were demonstrated, which indicated that little difference existed among the spectra of the three compounds. Therefore, CPM could not be identified by BACRA methodology.

3.3.2. The Discrimination of Chlorpheniramine Maleate by Characteristic Wavenumber Method. Different pretreatment methods were applied to the raw spectra of the three major 


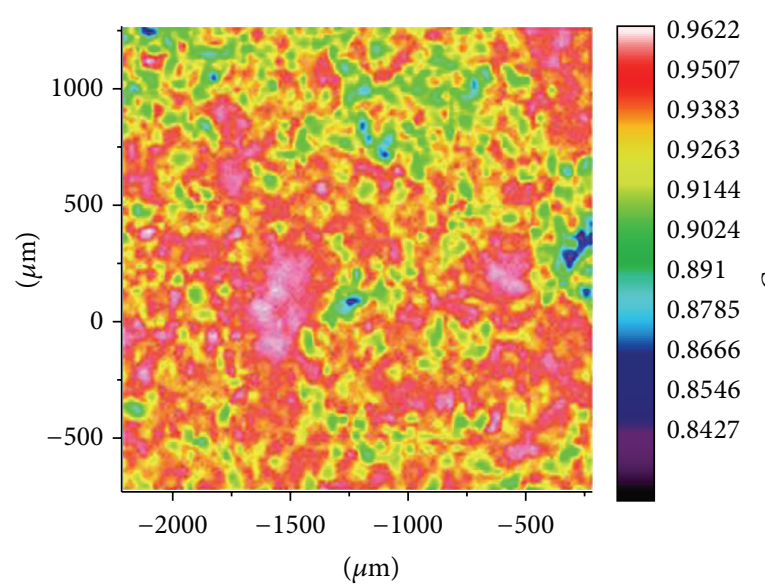

(a)

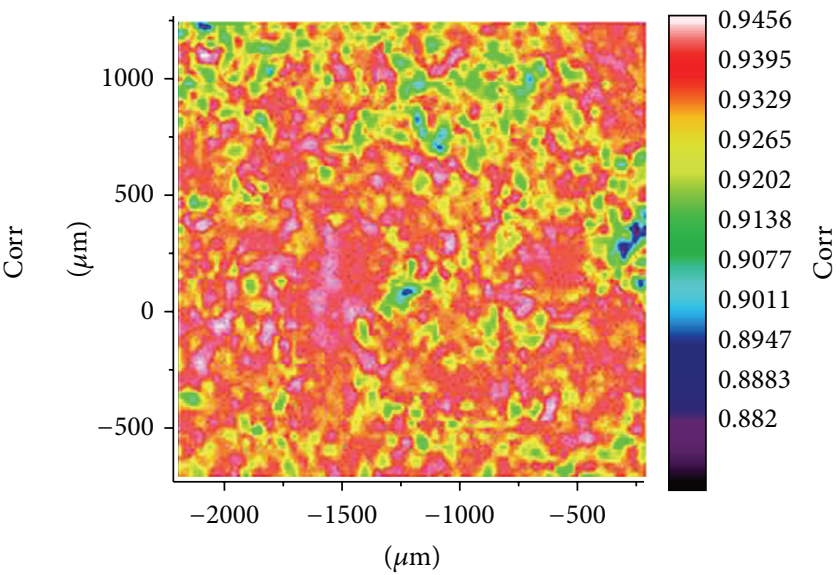

(b)

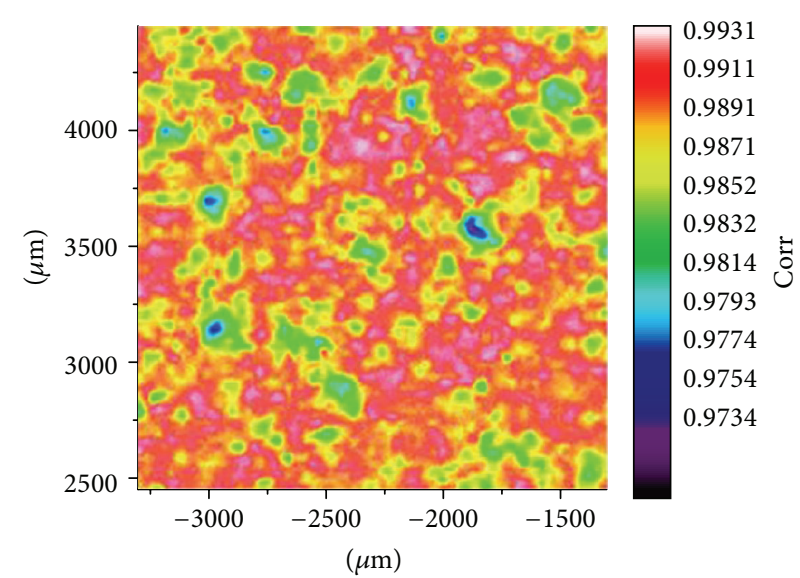

(c)

FIgURE 3: BACRA analysis for raw pure spectra and raw pure NIR image. (a) The correlation image from STA with pure CPM image, (b) the correlation image from MCC with pure CPM image, and (c) the correlation image from STA with pure MCC image.

ingredients. The spectra preprocessed by the combination of SG smoothing with a 9-point window and first derivative showed a distinct and sharp absorption feature for CPM at $6032 \mathrm{~cm}^{-1}$ (see Figure 2(c)). Therefore, the characteristic wavenumber method could be used to extract the CPM distribution.

The hyperspectral data obtained from the CPM tablet surface were unfolded and the spectra were preprocessed by SG smoothing and first derivative. Then, the characteristic images for CPM at $6032 \mathrm{~cm}^{-1}$ were obtained, as was shown in Figure 4. The red area (high first derivative of absorbance) represented the CPM distribution. CPM was distributed dispersedly in different regions. At the same time, there were some large red areas, which demonstrated the existence of CPM aggregations.

\subsection{Content Uniformity Assessment of CPM Tablets}

3.4.1. Surface Content Uniformity Assessment of CPM Tablets Using NIR-CI. It was difficult to extract spatial information on CPM according to the distribution of red area. It was possible to demonstrate the CPM distribution using binary images. A threshold limit for the first derivative of absorbance at $6032 \mathrm{~cm}^{-1}$ was set and binary images (Figure 5) were created corresponding to Figure 4 . The white area in the images exhibited the CPM distribution. The spectral absorption produced by all other compounds was taken as background (black area). In general, the CPM distribution on the tablet surface was dispersive, but inhomogeneous. Some large white areas were observed. This result was consistent with the consequence acquired from Figure 4.

However, human eyes are subjective and fuzzy, and the results might not be objective. In order to enable objective analysis, statistical measurement was performed to derive numerical information from Figure 5 and the results were displayed in Table 1. The distribution diameters of CPM were ranging from 35 to $108 \mu \mathrm{m}$ (Table 1). This result demonstrated the existence of CPM agglomerations on the surface of tablet. Through calculating the proportion of white region in the binary image, the CPM contents of different regions on the tablet surface were obtained. The relative standard deviation (RSD) value of CPM content was $12.37 \%>3.00 \%$, which meant that the CPM distribution on the tablet surface was not uniform. 


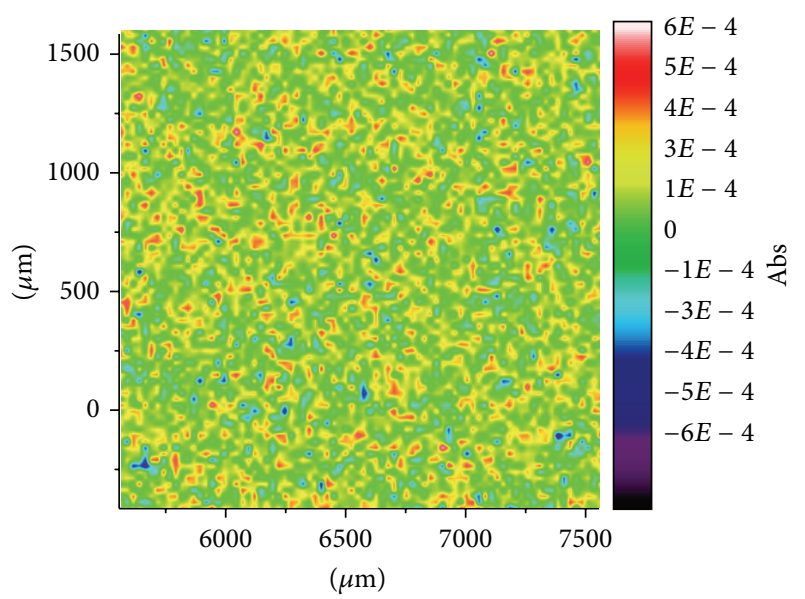

(a)

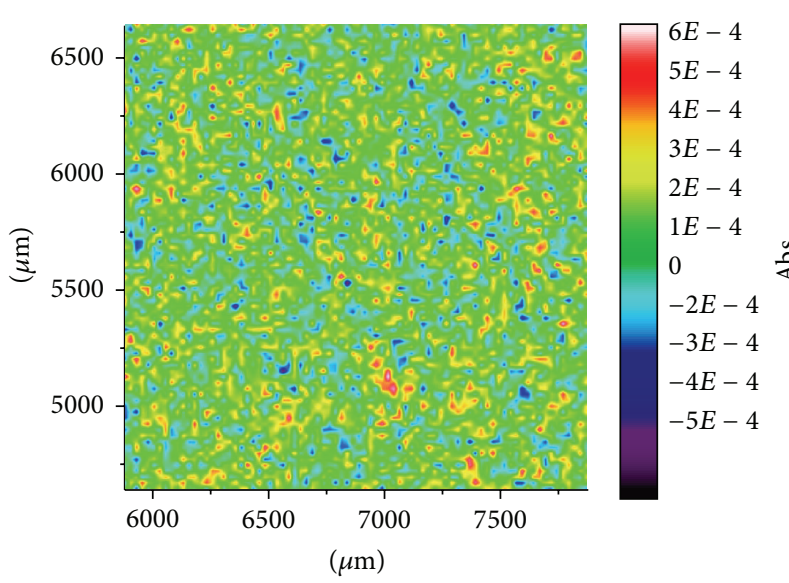

(c)

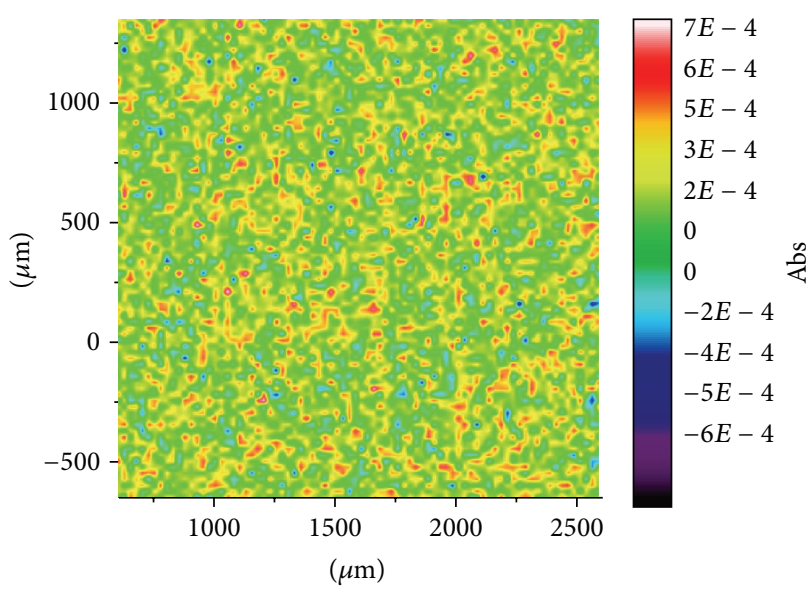

(b)

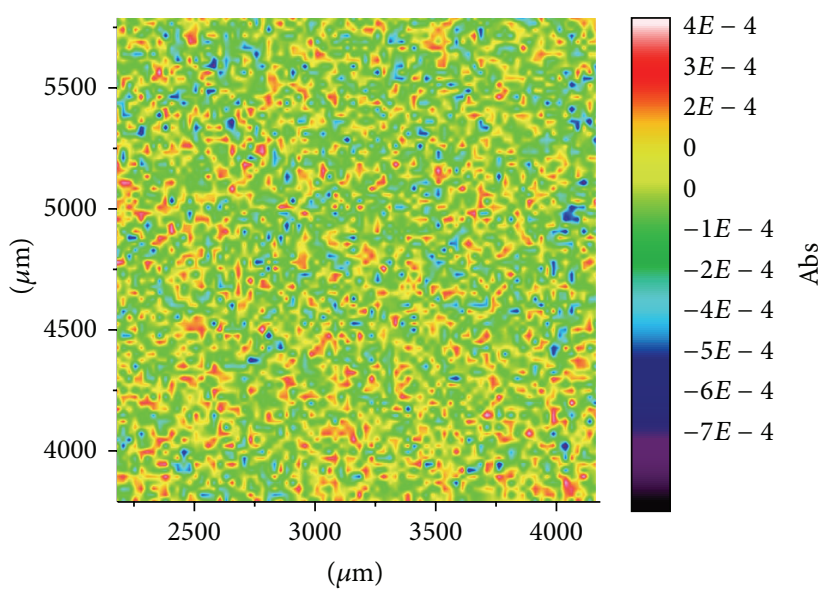

(d)

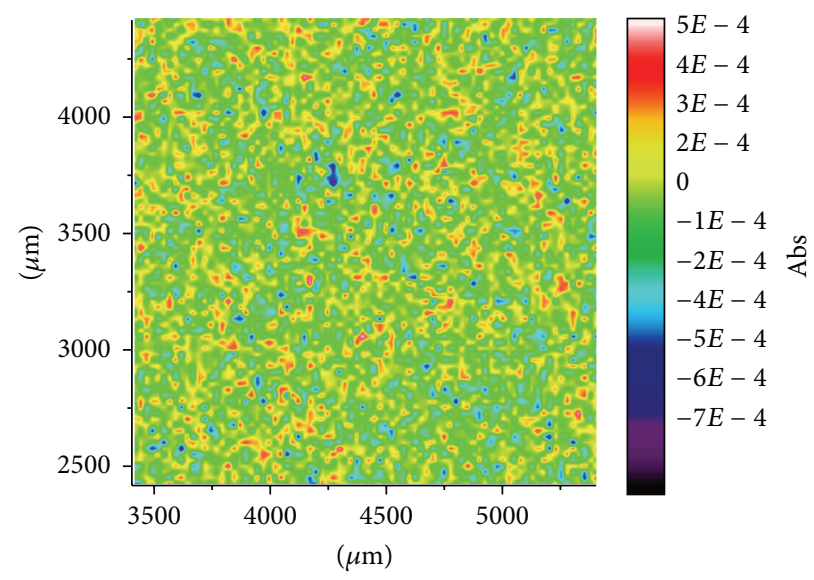

(e)

FIGURE 4: The characteristic images for CPM at $6032 \mathrm{~cm}^{-1}$, (a) upper-left region, (b) upper-right region, (c) lower-left region, (d) lower-right region, and (e) middle region on the tablet surface.

3.4.2. Volume Content Uniformity Assessment of CPM Tablets Using HPLC. HPLC was performed to assess volume content uniformity (VCU) of CPM in whole region and part region of the tablets. Firstly, ten CPM tablets were selected and each tablet was dissolved for VCU analysis. The evaluation index of volume content uniformity was 7.30 less than $15.00(A+1.8 S$, $A$ refers to absolute difference between labeled amount and average content and $S$ refers to standard deviation; Table 2), meaning the volume content uniformity of CPM satisfied the ChP (2010 edition) standard. 


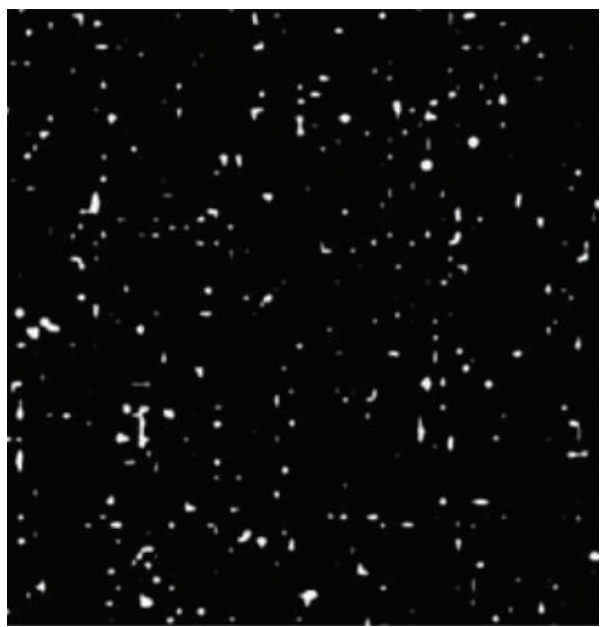

(a)

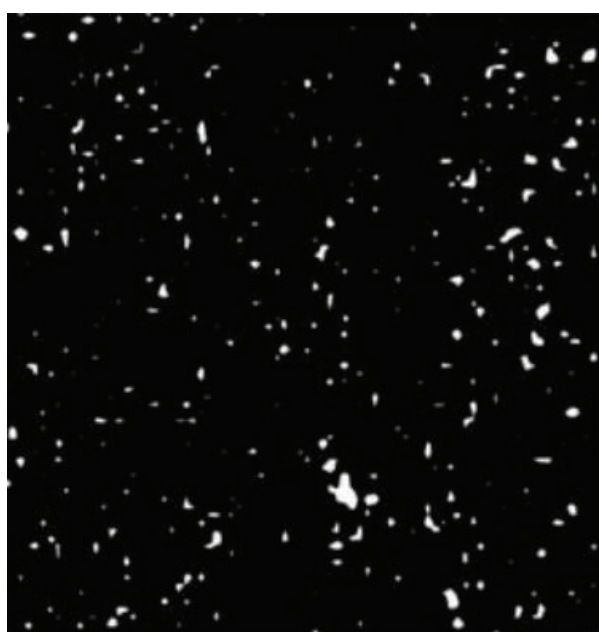

(c)

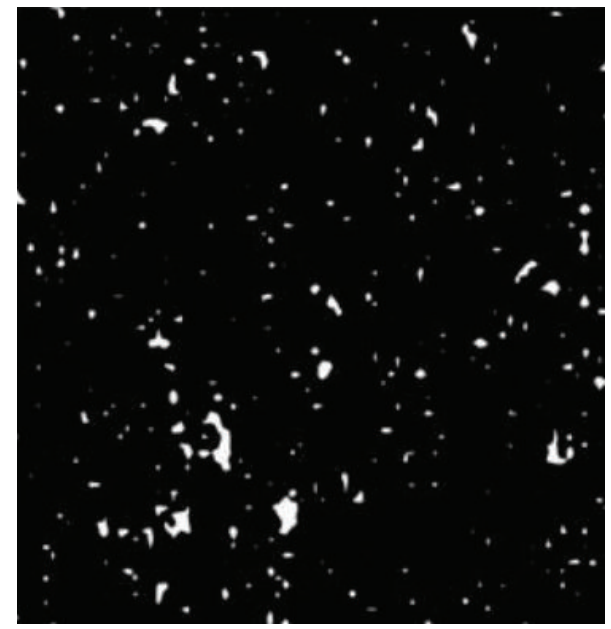

(b)

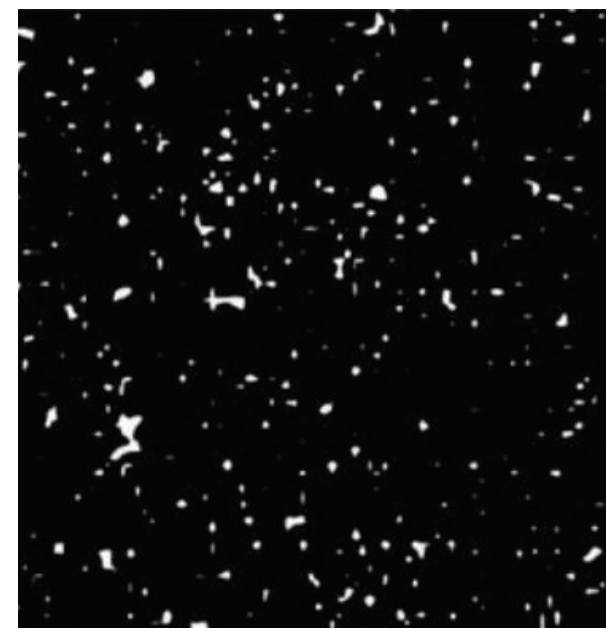

(d)

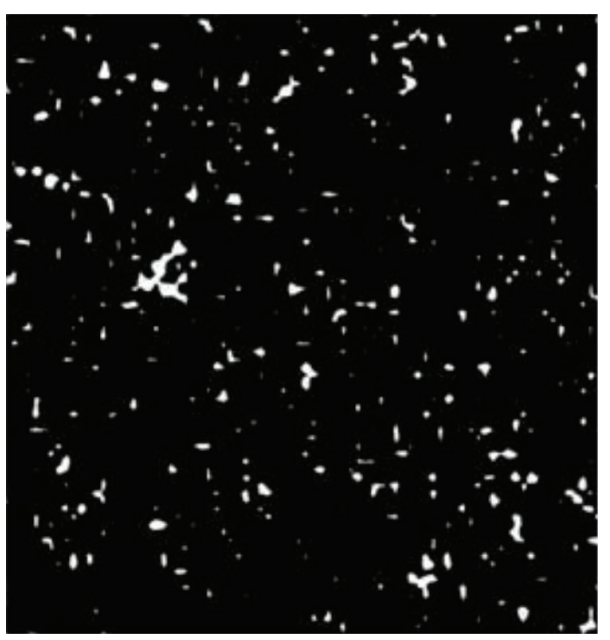

(e)

FIGURE 5: Binary images of CPM tablet. (a) Upper-left region, (b) upper-right region, (c) lower-left region, (d) lower-right region and, (e) middle region on the tablet surface. White region represented the cluster of CPM and black region represented the cluster of other components. 
TABLE 1: The consequence of statistical measurement by NIR-CI.

\begin{tabular}{lccc}
\hline Number & Range of diameters $(\mu \mathrm{m})$ & Average diameter $(\mu \mathrm{m})$ & Proportion of white region \\
\hline 1 & $37.78-60.41$ & 47.20 & $3.38 \%$ \\
2 & $64.07-108.11$ & 80.98 & $4.11 \%$ \\
3 & $49.73-78.77$ & 61.93 & $3.23 \%$ \\
4 & $35.22-68.29$ & 53.80 & $4.20 \%$ \\
5 & $39.79-64.94$ & 50.29 & $4.17 \%$ \\
\hline
\end{tabular}

${ }^{*}$ Number 1-5 represented upper-left region, upper-right region, lower-left region, lower-right region, and middle region on the tablet surface, respectively.

TABLE 2: Volume content uniformity assessment (whole samples) using HPLC.

\begin{tabular}{lcccccccccc}
\hline Number & 1 & 2 & 3 & 4 & 5 & 6 & 7 & 8 & 9 \\
\hline Peak area (mAU.s) & 706.8 & 738.2 & 743.8 & 755.5 & 799.3 & 718.5 & 720.3 & 737.1 & 728.0 & 719.5 \\
Content (mg) & 40.32 & 42.07 & 42.39 & 42.76 & 40.44 & 40.97 & 41.07 & 42.01 & 41.50 & 41.03 \\
$A+1.8 S$ & & & & & & & & & &
\end{tabular}

TABLE 3: Volume content uniformity assessment (quartered samples) using HPLC.

\begin{tabular}{|c|c|c|c|c|c|c|c|c|c|c|}
\hline Number & 1 & 2 & 3 & 4 & 5 & 6 & 7 & 8 & 9 & 10 \\
\hline Peak area (mAU.s) & 713 & 841.3 & 799.7 & 749.2 & 799.3 & 852.2 & 739.4 & 776.3 & 822.9 & 807.4 \\
\hline Content (mg) & 8.13 & 9.57 & 9.72 & 8.54 & 9.10 & 9.69 & 8.43 & 8.84 & 9.36 & 9.19 \\
\hline$A+1.8 S$ & \multicolumn{10}{|c|}{$19.44>15.00$} \\
\hline
\end{tabular}

Secondly, another ten tablets were chosen and each tablet was averaged into four pieces. One piece of each tablet was dissolved for HPLC determination. The evaluation index was $19.44>15.00$ (Table 3 ), which indicated that the volume content uniformity of CPM did not satisfy the requirement. Through the comparison of the two content uniformity tests, it illustrated that when volume content uniformity assessed by HPLC method conformed to the ChP (2010 edition) standard, it did not mean that the ingredients are distributed homogeneously in the sample.

The sample after NIR-CI scanning was divided into 4 pieces. The CPM content in each piece was determined by HPLC method. The volume contents of four pieces were $3.88 \%, 3.96 \%, 3.61 \%$, and $4.42 \%$, respectively. The RSD value was $8.52 \%>3.00 \%$. This result further demonstrated that the CPM distribution was not uniform.

3.5. Method Application on Commercial CPM Tablets. In addition, commercial CPM tablets (labeled CPM content: $4 \mathrm{mg}$, bought from local pharmacy) were also analyzed by NIR-CI and HPLC methods. The same data analysis approaches were used (detailed description and data were demonstrated in Supplementary Material available online at http://dx.doi.org/10.1155/2014/741246). The CPM content of different regions on the tablet surface was determined by NIR-CI and statistical measurement. The RSD value of CPM contents of different regions was $12.01 \%>3.00 \%$, indicating the inhomogeneous distribution of CPM on the tablet surface. The volume content uniformity of CPM in whole region and part region of the tablets was also determined by HPLC method. The evaluation indices were $12.69(<15.00)$ and 27.39 (>15.00), respectively. This result also demonstrated that ingredients might not be distributed homogeneously in the sample, even though the volume content uniformity determined by HPLC method conformed to the regulations, that is, the ChP (2010 edition).

\section{Conclusions}

This study demonstrated that NIR-CI was a rapid and nondestructive technique for discrimination of CPM and assessment of its SCU. Characteristic wavenumber method, binary image, and statistical measurement were applied to extract CPM distribution and assess its content uniformity on the tablet surface. Moreover, HPLC method was developed to assess volume content uniformity of CPM in whole region and part region of the tablets. Both CPM tablets produced from pilot scale by us and legitimate producer were investigated. The result revealed that the CPM distribution was not uniform on the sample surface, that is, the CPM contents of different regions on the tablet (made by ourselves) surface were determined by statistical measurement and the RSD value of CPM contents was $8.52 \%>3.00 \%$. Furthermore, the evaluation indices of VCU were 7.30 (whole samples) and 19.44 (quartered samples), respectively. The results indicated that the API might not be distributed homogeneously in the tablet, although the volume content uniformity of API assessed by HPLC fulfilled the ChP (2010 edition) standard. Through the comparison of content uniformity of CPM determined by NIR-CI and HPLC, respectively, it further indicated that a high degree of VCU did not imply a high degree of SCU of the samples. Therefore, HPLC is more accurate for volume content uniformity measurements, while NIR-CI is more suitable for surface content uniformity 
measurements and is suitable for the use in the quality control of pharmaceutical products.

\section{Conflict of Interests}

The authors declare that there is no conflict of interests regarding the publication of this paper.

\section{Acknowledgments}

Financial support of this work is from National Natural Science Foundation of China (no. 81303218), Doctoral Fund of Ministry of Education of China (20130013120006), Beijing University of Chinese Medicine Special Subject of Outstanding Young Teachers, Scientific Research Project of Beijing University of Chinese Medicine (2014JYBZZ-XS083), and Innovation Team Foundation of Beijing University of Chinese Medicine.

\section{References}

[1] World Health Organization, "Counterfeit Drugs: Guidelines for the development of measures to combat counterfeit drugs," 1999.

[2] R. Martino, M. Malet-Martino, V. Gilard, and S. Balayssac, "Counterfeit drugs: analytical techniques for their identification," Analytical and Bioanalytical Chemistry, vol. 398, no. 1, pp. 77-92, 2010.

[3] J. Yao, Y. Q. Shi, Z. R. Li, and S. H. Jin, "Development of a RP-HPLC method for screening potentially counterfeit antidiabetic drugs," Journal of Chromatography B, vol. 853, no. 1-2, pp. 254-259, 2007.

[4] W. C. Samms, Y. J. Jiang, M. D. Dixon, S. S. Houck, and A. Mozayani, "Analysis of alprazolam by DART-TOF mass spectrometry in counterfeit and routine drug identification cases," Journal of Forensic Sciences, vol. 56, no. 4, pp. 993-998, 2011.

[5] S. H. F. Scafi and C. Pasquini, "Identification of counterfeit drugs using near-infrared spectroscopy," Analyst, vol. 126, no. 12, pp. 2218-2224, 2001.

[6] O. Y. Rodionova and A. L. Pomerantsev, "NIR-based approach to counterfeit-drug detection," TrAC: Trends in Analytical Chemistry, vol. 29, no. 8, pp. 795-803, 2010.

[7] M. de Veij, P. Vandenabeele, K. A. Hall et al., "Fast detection and identification of counterfeit antimalarial tablets by Raman spectroscopy," Journal of Raman Spectroscopy, vol. 38, no. 2, pp. 181-187, 2007.

[8] C. Eliasson and P. Matousek, "Noninvasive authentication of pharmaceutical products through packaging using spatially offset Raman spectroscopy," Analytical Chemistry, vol. 79, no. 4, pp. 1696-1701, 2007.

[9] A. Palou, J. Cruz, M. Blanco, J. Tomàs, J. de los Ríos, and M. Alcalà, "Determination of drug, excipients and coating distribution in pharmaceutical tablets using NIR-CI," Journal of Pharmaceutical Analysis, vol. 2, no. 2, pp. 90-97, 2012.

[10] H. Ma and C. A. Anderson, "Characterization of pharmaceutical powder blends by NIR chemical imaging," Journal of Pharmaceutical Sciences, vol. 97, no. 8, pp. 3305-3320, 2008.

[11] J. Cruz and M. Blanco, "Content uniformity studies in tablets by NIR-CI," Journal of Pharmaceutical and Biomedical Analysis, vol. 56, no. 2, pp. 408-412, 2011.
[12] J. Dubois, J. Wolff, J. K. Warrack, J. Schoppelrei, and E. N. Lewis, "NIR chemical imaging for counterfeit pharmaceutical products analysis," Spectroscopy, vol. 22, no. 2, pp. 40-50, 2007.

[13] A. A. Gowen, C. P. O'Donnell, P. J. Cullen, and S. E. J. Bell, "Recent applications of chemical imaging to pharmaceutical process monitoring and quality control," European Journal of Pharmaceutics and Biopharmaceutics, vol. 69, no. 1, pp. 10-22, 2008.

[14] C. Gendrin, Y. Roggo, and C. Collet, "Pharmaceutical applications of vibrational chemical imaging and chemometrics: a review," Journal of Pharmaceutical and Biomedical Analysis, vol. 48, no. 3, pp. 533-553, 2008.

[15] D. C. Hinz, "Process analytical technologies in the pharmaceutical industry: the FDA's PAT initiative," Analytical and Bioanalytical Chemistry, vol. 384, no. 5, pp. 1036-1042, 2006.

[16] L. X. Yu, "Pharmaceutical quality by design: product and process development, understanding, and control," Pharmaceutical Research, vol. 25, no. 4, pp. 781-791, 2008.

[17] J. Kirkegaard, C. Secher, and N. Mygind, "Effect of the $\mathrm{H}_{1}$ antihistamine chlorpheniramine maleate on histamine-induced symptoms in the human conjunctiva," Allergy, vol. 37, no. 3, pp. 203-208, 1982.

[18] C. Ravn, E. Skibsted, and R. Bro, "Near-infrared chemical imaging (NIR-CI) on pharmaceutical solid dosage formsComparing common calibration approaches," Journal of Pharmaceutical and Biomedical Analysis, vol. 48, no. 3, pp. 554-561, 2008.

[19] S. Šašić, "An in-depth analysis of Raman and near-infrared chemical images of common pharmaceutical tablets," Applied Spectroscopy, vol. 61, no. 3, pp. 239-250, 2007.

[20] Z. S. Wu, O. Tao, X. X. Dai, M. Du, X. Y. Shi, and Y. J. Qiao, "Monitoring of a pharmaceutical blending process using near infrared chemical imaging," Vibrational Spectroscopy, vol. 63, pp. 371-379, 2012.

[21] J. Huang, H. Wium, K. B. Qvist, and K. H. Esbensen, "Multi-way methods in image analysis-relationships and applications," Chemometrics and Intelligent Laboratory Systems, vol. 66, no. 2, pp. 141-158, 2003.

[22] M. Vidal and J. M. Amigo, "Pre-processing of hyperspectral images. Essential steps before image analysis," Chemometrics and Intelligent Laboratory Systems, vol. 117, pp. 138-148, 2012. 

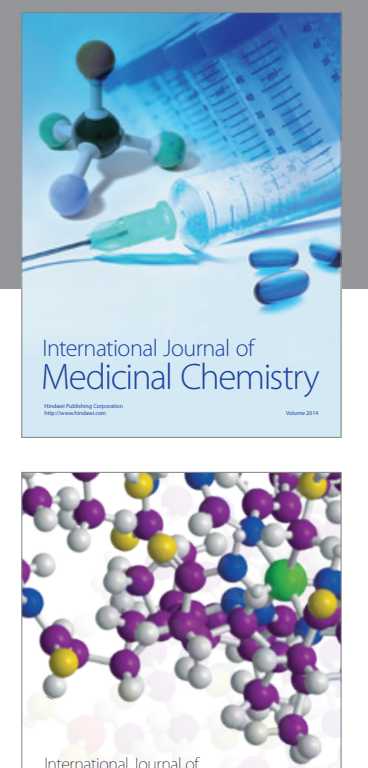

\section{Carbohydrate} Chemistry

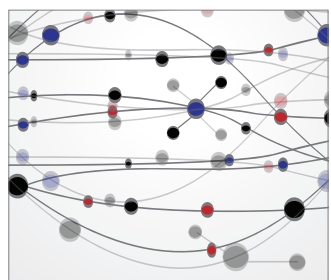

The Scientific World Journal
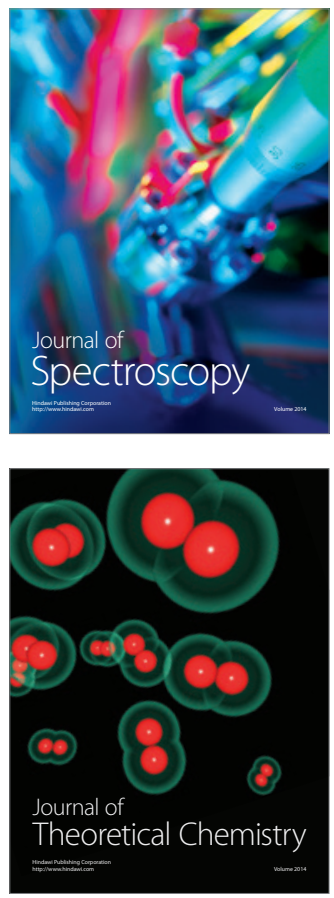
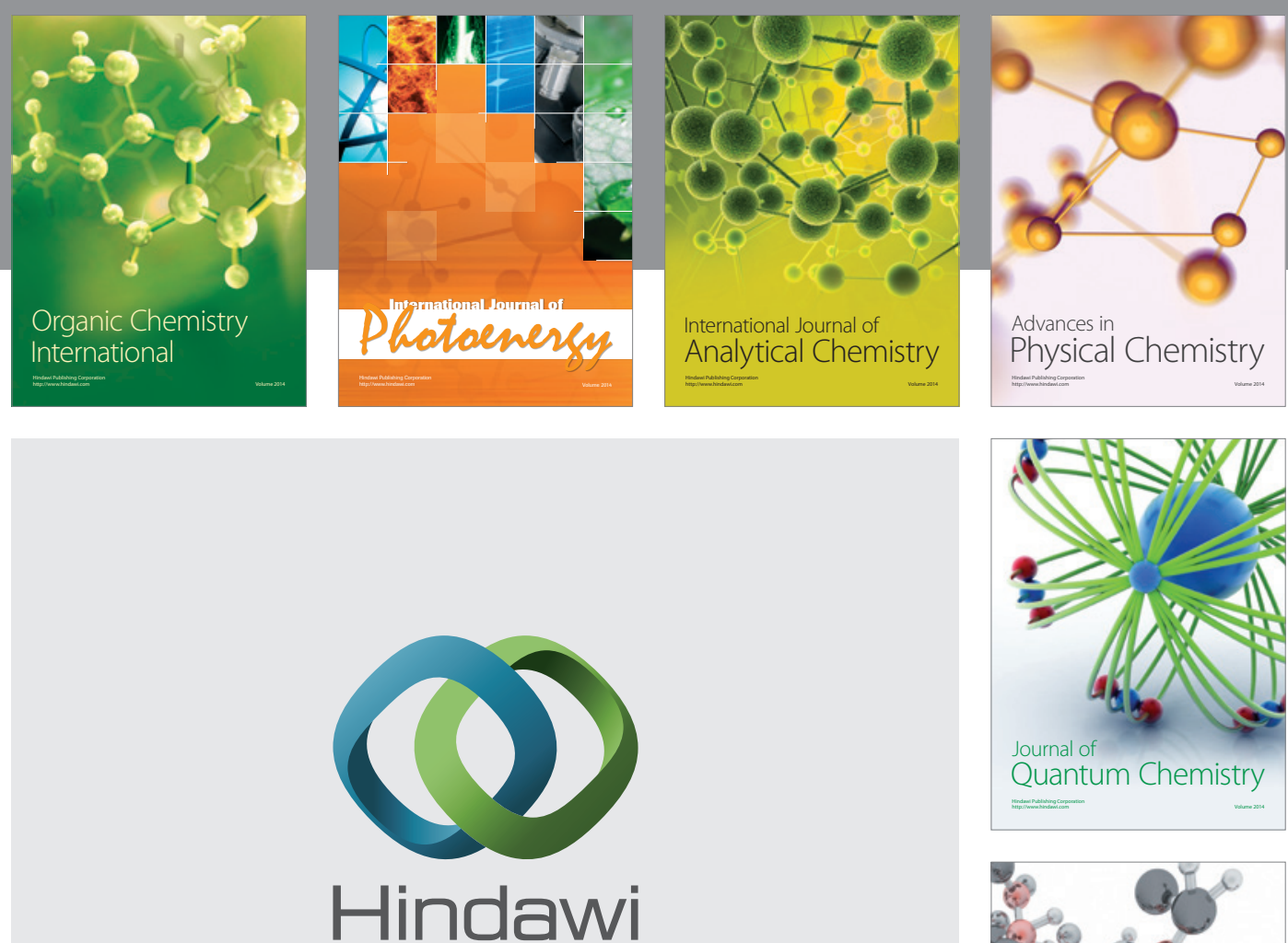

Submit your manuscripts at

http://www.hindawi.com

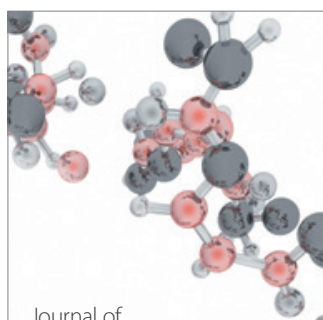

Analytical Methods

in Chemistry

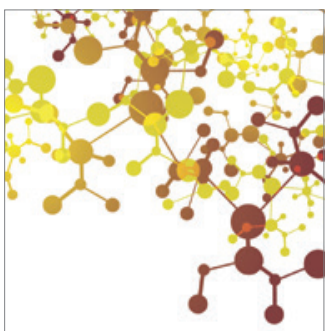

Journal of

Applied Chemistry

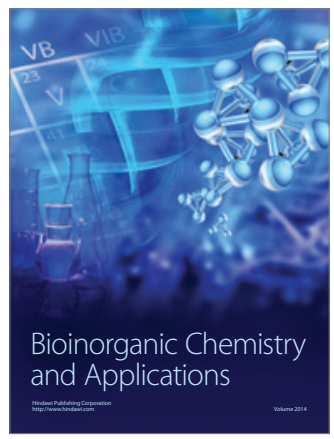

Inorganic Chemistry
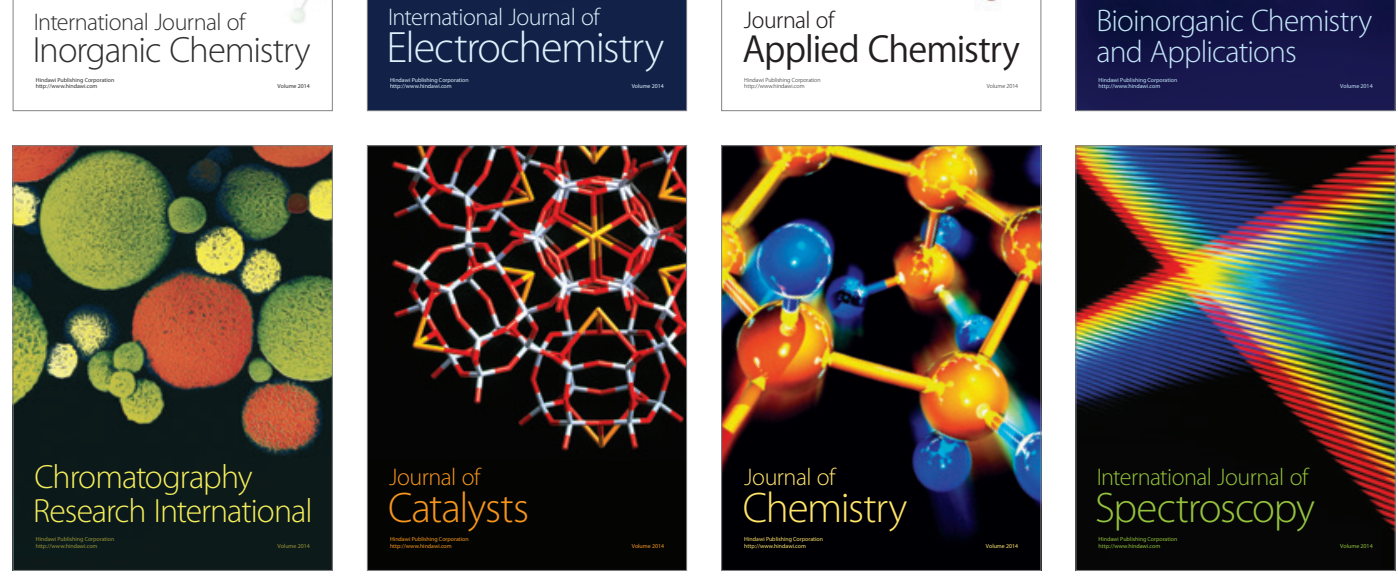